\title{
Levantamiento estratigráfico detallado para el miembro Shale de Bambucá en dos secciones de la subcuenca de Neiva
}

\author{
Stratigraphic detailed survey for Shale of Bambucá \\ member in two sections of Neiva subbasin
}

\author{
Roberto Vargas Cuervo', Carlos Andrés Pérez Badillo² y Carlos Iván Díaz Quimbaya ${ }^{3}$
}

\begin{abstract}
Resumen
El estudio de los ambientes de sedimentación, proporcionan a la industria del petróleo el conocimiento sobre los sucesos sedimentarios ocurridos y que llevaron a cabo la existencia de las formaciones productoras, generadoras y sello; en este documento son estudiadas las rocas generadoras del miembro Shale de Bambucá, perteneciente la formacion Villeta en la Subcuenca de Neiva; Valle Superior del Magdalena (VSM). Para el desarrollo de la investigación se levantaron columnas estratigráficas en dos sitos, La Tribuna y Los Yuyos; en los cuales se observan secciones predominantes de lodolitas con características que las identifican en ambientes de plataforma, fuera de la influencia del oleaje. Las columnas fueron analizadas y divididas en secuencias y parasecuencias, enfocadas en los cambios eustáticos que a su vez determinaron la depositación de sedimento y materia orgánica.
\end{abstract}

Palabras clave: Shale de Bambucá; Estratigrafia secuencial; Curva eustática.

\begin{abstract}
The study of sedimentary environment, provide to the petroleum industry the knowledge about the sedimentary events occurred and how they made (it create) the production formations, source rocks and trap rocks; in this paper the source rock from Bambucá Shale member, it belongs to Villeta formation in the Neiva subbasin; Upper Magdalena Valley (UMV). In order to develop an investigation stratigraphic colum in two sites, La Tribuna and Los Yuyos; where are observed predominant sections of mudstone with identifying characteristics of platform environments, out to the influenfe of the waves. The columns were analyzed and divided into sequences and parasequences, focused in eustatic changes which determined the deposition of sediment and organic matter.
\end{abstract}

Keywords: Bambucá Shale; Sequence stratigraphy; Eustatic curve.

1 - GeólogoMsc. Docente Universidad Surcolombiana, Neiva. Avenida Pastrana Borrero carrera 1a Neiva, roberto.vargas@usco.edu.co

2 - Ingeniero de Petróleos. Universidad Surcolombiana, Neiva.Avenida Pastrana Borrero carrera la Neiva, capb_9008@hotmail.com

3 - Ingeniero de Petróleos. Universidad Surcolombiana, Neiva. Avenida Pastrana Borrero carrera 1a Neiva, diazc3015@gmail.com 


\section{Introducción}

Debido a los grandes requerimientos de energía que actualmente demanda el mundo, los yacimientos de hidrocarburos, cada vez son más escasos y difíciles de alcanzar. En épocas pasadas se creía que los reservorios se encontraban exclusivamente en un tipo de rocas sedimentarias, pero existen alrededor del mundo varios ejemplos con los que se puede demostrar que las rocas pelíticas aparte de ser las rocas generadoras de hidrocarburos, bajo ciertas condiciones, pueden albergar cantidades considerables de gas y aceite. Por tal razón es importante identificar las características petrológicas de las rocas, para determinar la existencia de hidrocarburos no convencionales asociados a shale, además de conocer la existencia de secuencias de rocas lutíticas en la zona.

El presente trabajo representa la investigación sobre las rocas pertenecientes a la formación Villeta, realizando análisis de los diferentes tipos de litología, ambientes sedimentarios que propiciaron la depositación de minerales y materia orgánica generadora de hidrocarburos con kerógeno tipo II (Atlas 2000) y contenido de materia organica (TOC) de 1 a 4\% (Sarmiento, L.F. \& Rangel A., 2003), unidades litoestratigráficas que identifiquen la ubicación y geología estructural para la elaboración del levantamiento estratigráfico del miembro Bambucá. Con esto se determinaron las curvas eustáticas, representativas de las variaciones del nivel del mar y el ambiente sedimentario del depósito.

En los afloramientos identificados en La Tribuna y Los Yuyos fueron levantados teniendo en cuenta la cartografia realizada por el instituto geográfico Agustin Codazzi en los espaciomapas 323 (Ferreira et al., 2002) y 344 (Marquinez G., 2002), respectivamente; en los lugares de observación predominan lodolitas de color oscuro, con laminación plano paralela, que sugiere una precipitación de minerales y materia orgánica; estas características corresponden según la estratigrafía secuencial, al límite de secuencias (Van Wagoner et al. 1988) como rocas acumuladas en ambientes deposicionales de plataforma interna.

Este documento acoge la nomenclatura de la industria petrolera, dividiendo la formación Villeta en cuatro miembros: Aico Chert, La Luna, Bambucá y Tetuán, dada la inconsistencia con la litología propuesta por INGEOMINAS (Peña J., et al., 2002), donde la formación se divide en dos miembros: Hondita y Lomagorda.

\section{Metodología}

Para el desarrollo del presente trabajo fueron seleccionados dos sectores de la Subcuenca de Neiva, la Tribuna es un centro de educación ambiental de Ecopetrol y el sector de los Yuyos, que se encuentra en la cuenca del rio Yaguaracito en Tesalia; ambos fueron escogidos especialmente debido a los afloramientos de lutitas existentes en el área. Se realiza un enfoque especial al miembro Shale de Bambucá con el objetivo de definir litoestratigraficamente las unidades (Facies) que lo conforman, definir el ambiente sedimentario en el que se depositó la materia orgánica y las estructuras sedimentarias generadoras de los hidrocarburos. El estudio y reconocimiento de las rocas pertenecientes a la formación Villeta en la subcuenca de Neiva dan un enfoque adecuado para la extracción de hidrocarburos asociados a Shales. Estratigráficamente en el área aflora toda la cobertura productiva de la Subcuenca de Neiva representada por las formaciones Caballos, la formación Villeta con el miembro Shale de Bambucá, la formación Monserrate y formación Guaduala.

La nomenclatura utilizada para la elaboración de las columnas estratigráficas en este proyecto es la utilizada por las compañías petroleras que operan en la zona del Valle Superior del Magdalena, la cual tiene ciertas diferencias con la utilizada por INGEOMINAS (Tabla 1). 


\begin{tabular}{|c|c|c|c|c|c|c|}
\hline \multicolumn{3}{|r|}{ EDAD } & \multicolumn{2}{|c|}{$\begin{array}{c}\text { NOMENCLATURA } \\
\text { EMPLEADA EN LA } \\
\text { INDUSTRIA PETROLERA }\end{array}$} & \multicolumn{2}{|c|}{$\begin{array}{l}\text { NOMENCLATURA } \\
\text { EMPLEADA POR } \\
\text { INGEOMINAS } \\
\end{array}$} \\
\hline \multicolumn{2}{|c|}{ ERA } & PERIODO & GRUPO & FORMACIÓN & GRUPO & FORMACIÓN \\
\hline \multirow{12}{*}{ 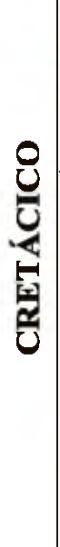 } & \multirow{4}{*}{ 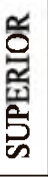 } & \multirow{4}{*}{$\begin{array}{l}\text { Maastrichtiano } \\
\text { Campaniano }\end{array}$} & \multirow{4}{*}{ Monserrate } & $\mathbf{K} 1$ & \multirow{4}{*}{ K III } & \multirow{2}{*}{$\begin{array}{c}\text { Buscavidas } \\
\text { La Tabla }\end{array}$} \\
\hline & & & & $\mathrm{K} 2$ & & \\
\hline & & & & K3 & & \multirow{2}{*}{ Olini } \\
\hline & & & & K4 & & \\
\hline & \multirow{8}{*}{$\frac{\mathscr{2}}{\frac{2}{2}}$} & \multirow{4}{*}{$\begin{array}{c}\text { Santoniano } \\
\text { Albiano }\end{array}$} & \multirow{4}{*}{ Villeta } & Aico Chert & \multirow{4}{*}{ K II } & \multirow{2}{*}{ Loma Gorda } \\
\hline & & & & La Luna & & \\
\hline & & & & Shale de Bambucá & & \multirow{2}{*}{ Hondita } \\
\hline & & & & Calizas de Tetuán & & \\
\hline & & \multirow{2}{*}{ Albiano } & \multirow{3}{*}{ Caballos } & Superior & \multirow{4}{*}{ K I } & \multirow{3}{*}{ Caballos } \\
\hline & & & & Medio & & \\
\hline & & \multirow{2}{*}{ Aptiano } & & Inferior & & \\
\hline & & & & Yaví & & Yaví \\
\hline
\end{tabular}

Tabla 1. Sección de la columna estratigráfica utilizada en este estudio, con la relación entre las nomenclaturas de la industria petrolera e Ingeominas.

\subsection{Localización Geográfica}

\section{La Tribuna}

La Tribuna(Figura 1), se localiza en estribaciones del piedemonte de la cordillera central, en la cuenca del río Magdalena y la microcuenca de la quebrada El Neme, a una elevación de 925 metros sobre el nivel del mar, en territorio de la vereda Tamarindo, en el norte del Departamento del Huila, aproximadamente a 28 Kilómetros del municipio de Neiva; posee un área aproximada de 128 hectáreas manejado inicialmente por la fundación Hocol y actualmente por Ecopetrol, siendo un área que inicialmente fue de actitud ganadera con alto deterioro erosivo. Cuenta con una amplia caracterización de la biosfera, flora, fauna y suelos. Geológicamente la sucesión estratigráfica más común en la Tribuna está representada por una secuencia monótona de shales negros pertenecientes al miembro Bambucá de la formación Villeta.

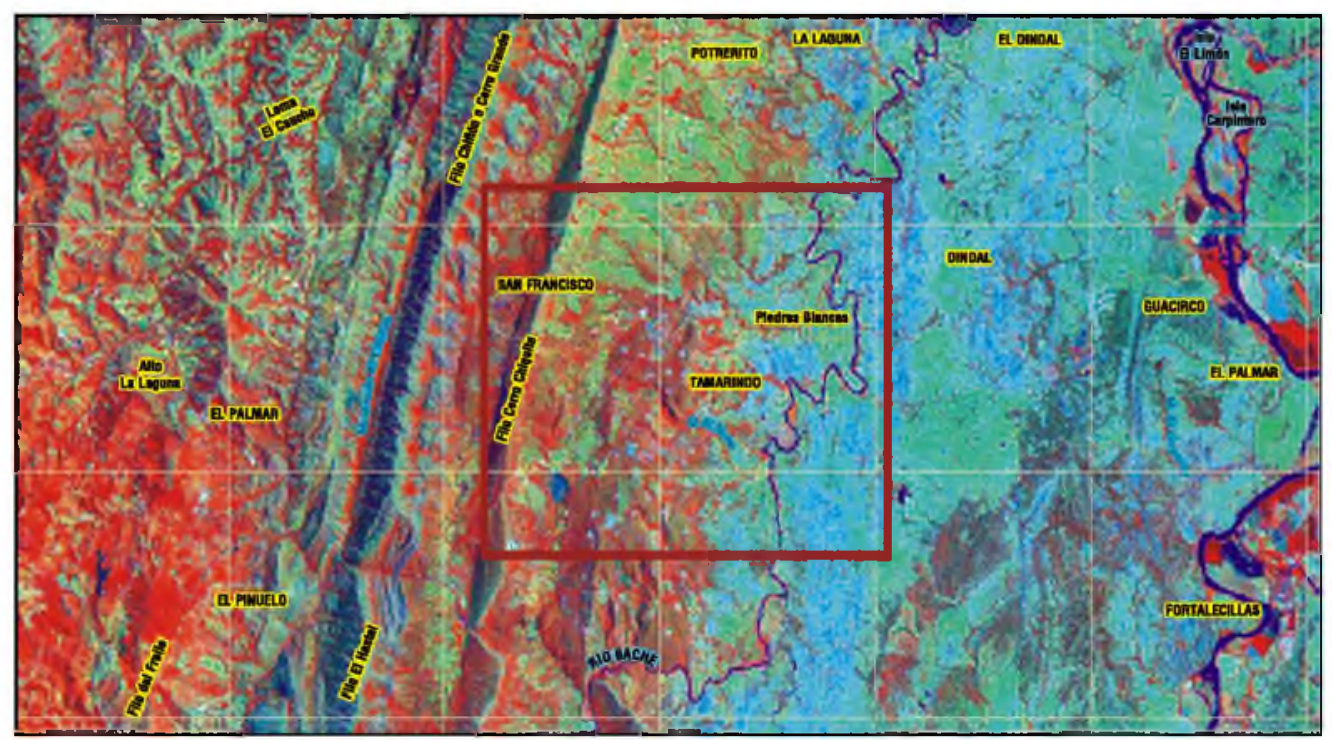

Figura 1. Modelo de elevación digital del área central de la Tribuna donde se observa la morfología y alineamientos estructurales. Sección del Espaciomapa 323, SIGAC. 


\section{Los Yuyos}

Se localiza en la vereda Yuyos (Figura 2), municipio de Tesalia departamento del Huila, se llega a ella por la carretera pavimentada de $100 \mathrm{Km}$. que une a Neiva con Tesalia y se sigue $13 \mathrm{Km}$ por la carretera Tesalia - Pacarní hasta la escuela Hugo Escobar Guerrero, de donde parte un carreteable de acceso a las vereda los Yuyos. El clima es templado, con temperatura promedia de $24^{\circ} \mathrm{C}$. La vegetación es la típica de la región de bosque húmedo tropical; con carencia de bosque el cual en su mayoría ha desaparecido debido a la intervención antrópica, que ha dejado en la mayoría del área praderas (potreros) dedicados al pastoreo de vacunos.

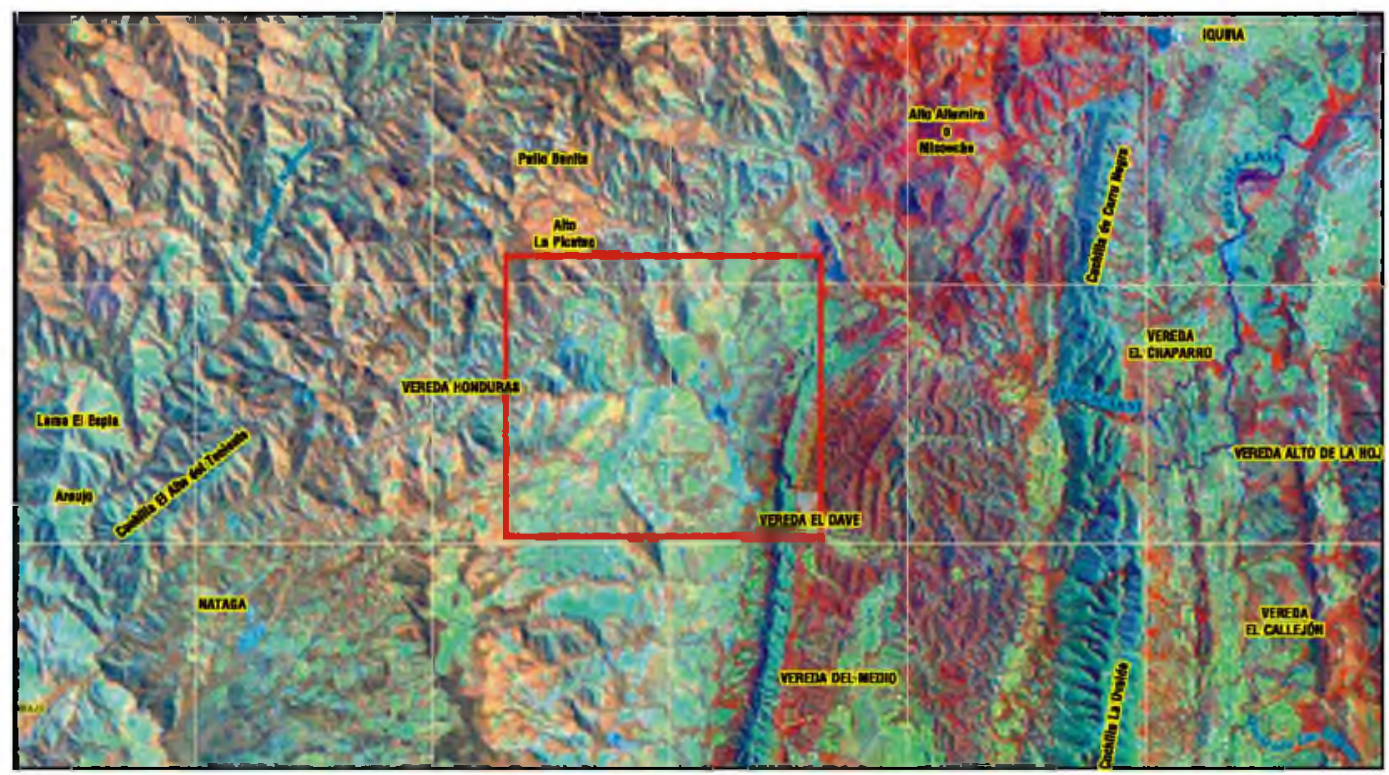

Figura 2. Modelo de elevación digital del área central de Los Yuyos donde se observa la morfología y alineamientos estructurales. Sección del Espaciomapa 344, SIGAC.

\subsection{Procedimiento para muestreo}

Estudios litológicos, estratigráficos estructurales detallados permiten entender la génesis y los procesos geológicos ocurridos en la secuencias estratigráficas, para realizar estos trabajos es necesario incluir como herramienta metodológica el muestreo de las rocas sedimentarias. El muestreo es efectuado mediante el empleo de alguna técnica especifica en cada una de las secciones aflorantes, considerándose como la primera etapa de todo trabajo sedimentológico, estructural y geológico.

El muestreo que se llevó a cabo de los depósitos sedimentarios para los fines de estudios detallados de laboratorio dependerá particularmente de los siguientes parámetros.

El levantamiento se realizó a escala detallada (1:100), mediante el método de la vara de Jacob y por cálculo de espesores aparentes, teniendo en cuenta todos los parámetros litológicos, texturales, petrofísicos; con el objetivo de aplicar la información obtenida del levantamiento estratigráfico a la estratigrafía secuencial, tomando como prioridad las estructuras sedimentarias presentes, los cuales dan indicaciones de la actividad ambiental dominante en el momento de la sedimentación, siendo la base para interpretar y aplicar la estratigrafía secuencial. En algunas áreas de estudio fue necesaria la aplicación de balanceo de secciones con el fin de no repetir secuencias perjudicadas por fallas de cabalgamiento de acuerdo al método geométrico.

\subsection{Sistema deposicional}

Según los análisis realizados a los parámetros de litología y estructuras sedimentarias, las estructuras internas tales como laminación, forma y espesor de las capas, se pudo realizar la curva eustática, permitiendo describir las litofacies con las que se logra determinar los diferentes tipos de sistemas deposicionales o system tract. En la Figura 3 vemos en una línea, el área de despositación que tuvo el miembro Shale de Bambucá, posicionándolo en un ambiente de plataforma media a externa y un poco de interna, 
donde hay una influencia del oleaje leve, propiciando una decantación de sedimentos y material orgánico de forma pareja y continua.

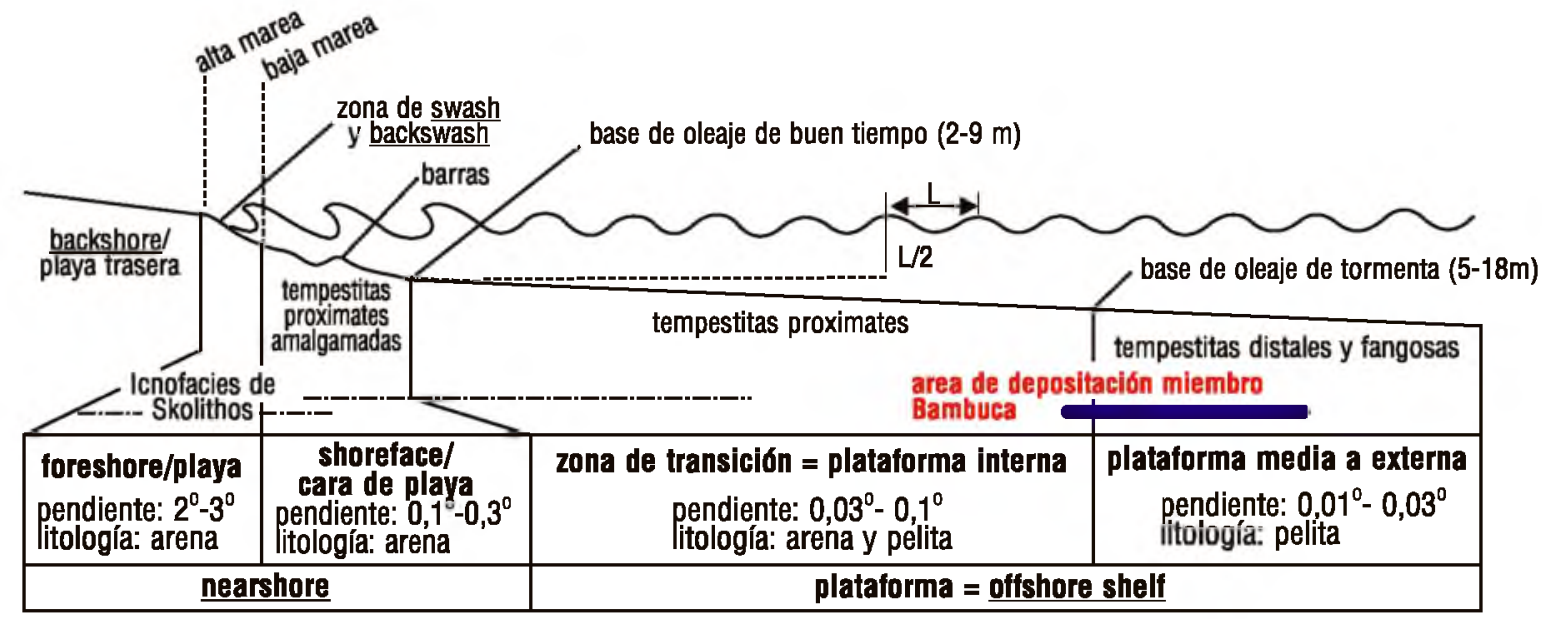

Figura 3. Ambientes de sedimentación. Zona de depositación para el miembro Shale de Bambucá en la Subcuenca de Neiva representada en la línea color azul.

Lo anterior teniendo en cuenta los órdenes cíclicos, que determinan el acomodamiento de las secuencias de depositación (Figura 4).

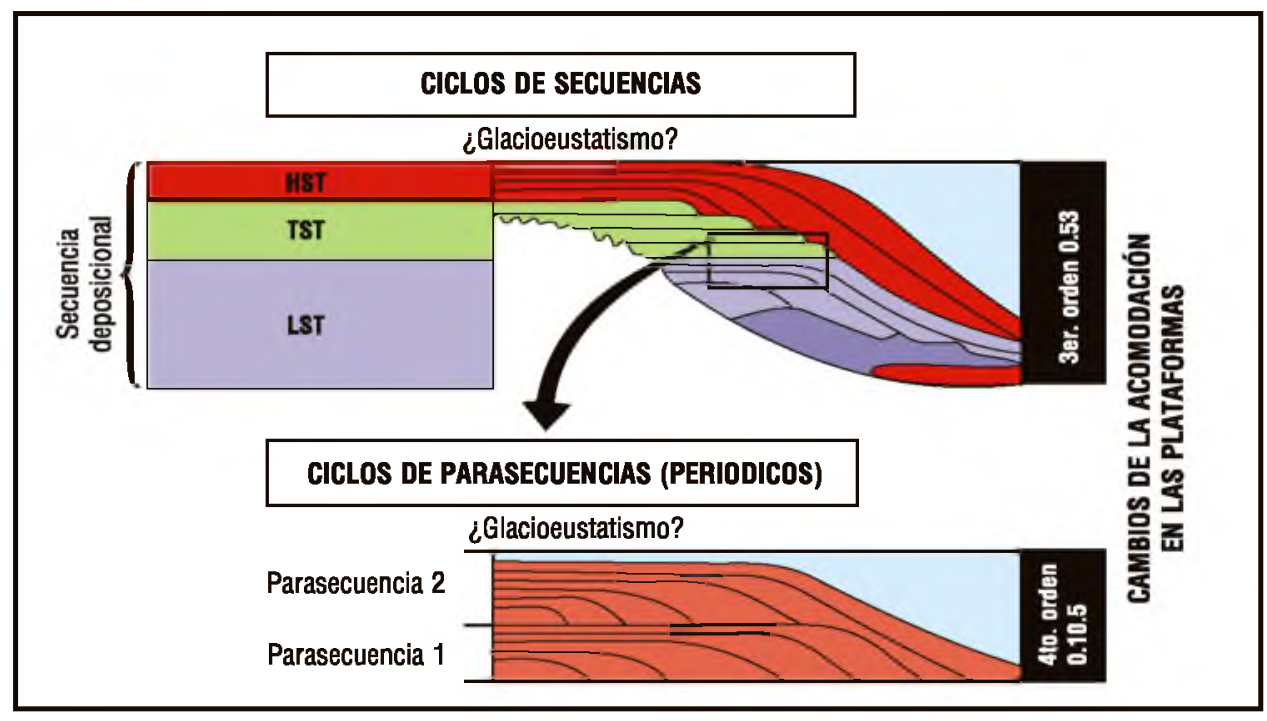

Figura 4. Dos órdenes de los ciclos estratigráficos. Sección tomada de Duvel et al (1992).

\section{Resultados}

En esta sección se representan las gráficas de poligonales y columnas estratigráficas obtenidas a partir del análisis de los datos y muestras tomado en campo:

\section{La Tribuna}

De acuerdo a los datos recogidos en campo, la poligonal abierta levantada de tope a base para el área de La Tribuna tiene un recorrido de 1195.9 metros por la microcuenca de la quebrada El Neme con 9 segmentos, levantando una columna estratigráfica con 165 metros de espesor (Figura 5). En ella son característicos los afloramientos de lutitas con intercalaciones de arcillolitas, limolitas y areniscas de grano fino a muy fino.

Los afloramientos en la sección de La Tribuna fueron enumerados en segmentos, para la elaboración de la 


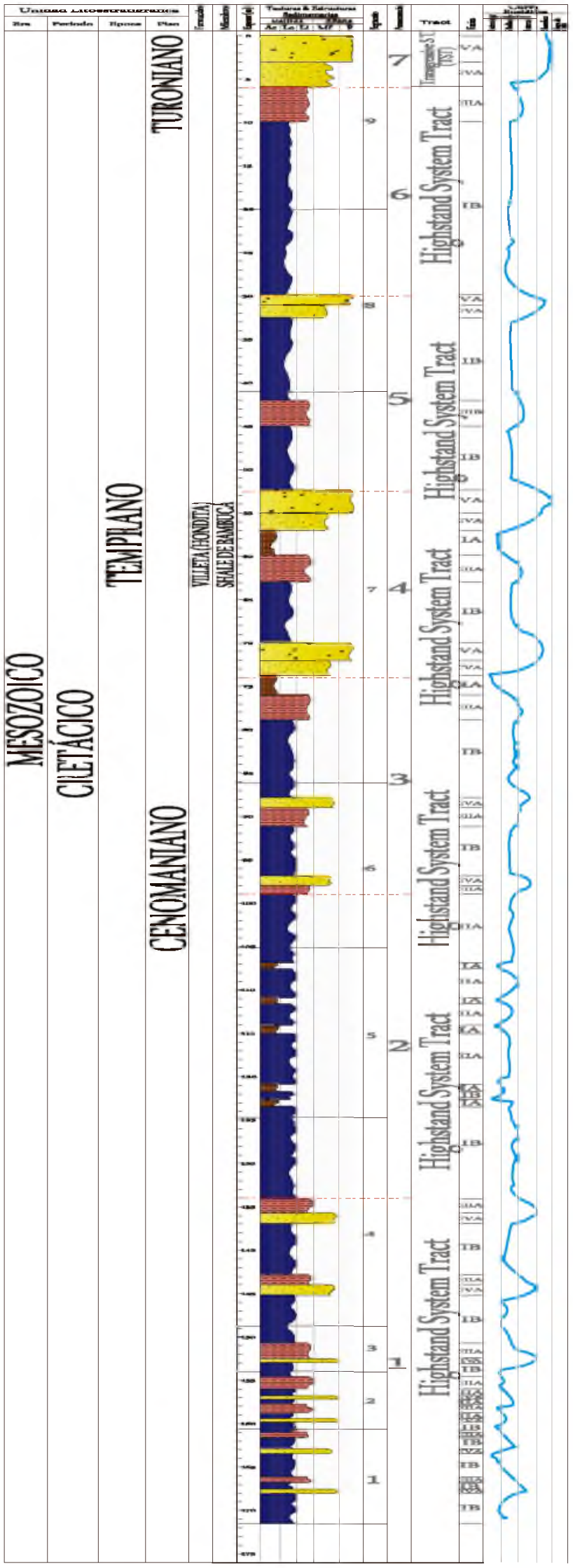

Figura 5.

Columna estratigráfica para La Tribuna. columna estratigráfica y esta fue dividida luego en System Tract y parasecuencias, que representan un lapso de tiempo en el que los depósitos describen un patrón de acomodación representativo de ambientes de plataforma.

E1 levantamiento estratigráfico, la caracterización del sistema deposicional y la recolección de muestras relacionan al miembro Shale de Bambucá en el área de la Tribuna como una cuenca sedimentaria con potencial hidrocarburífero, aunque hace falta concretar los estudios para determinar contenidos de materia orgánica TOC con la prueba de Pyrolisis.

\section{Transgressive System Tract}

Conformado litológicamente por margas, calizas y delgados niveles arenosos que representan un cambio en el sistema deposicional de plataforma interna a shoreface indicando que el nivel del mar es menos profundo haciendo que los ambientes de sedimentación sean menos tranquilos.

Parasecuencia 7. Con un espesor de 6 metros en donde se presenta una secuencia de rocas clasticas y bioquimicas . Hacia la base se presenta un paquete de lodolitas calcareas, lodolitas carbonosas y margas de color gris claro. Esta parasecuencia representa una sucesión progradante de ambientes del shoreface medio a la línea de costa.

\section{Highstand System Tract}

Manifestada como una superficie de baja energía generada durante un ascenso relativo del nivel del mar. De acuerdo al análisis litológico y las estructuras sedimentarias presentes la sección estratigráfica correspondiente al HST lo cual refleja un ascenso del nivel del mar y por ende una profundización en la cuenca.

Parasecuencia 6. Comprende un espesor de 24 metros, de lodolitas de color negro, presentan localmente a través de sus planos de estratificación muestras de hidrocarburos. Esta parasecuencia abarca ambientes de plataforma interna.

Parasecuencia 5. Con un espesor de 22 metros, la litología y estructuras sedimentarias de las lodolitas presentes en esta parasecuencia reflejan una variación desde ambientes de plataforma externa a shoreface.

Parasecuencia 4. Se presenta una secuencia de arcillolitas y lodolitas con un espesor de 21.5 metros. El análisis facial a esta parasecuencia refleja una variación desde ambientes de plataforma externa a shorface. 


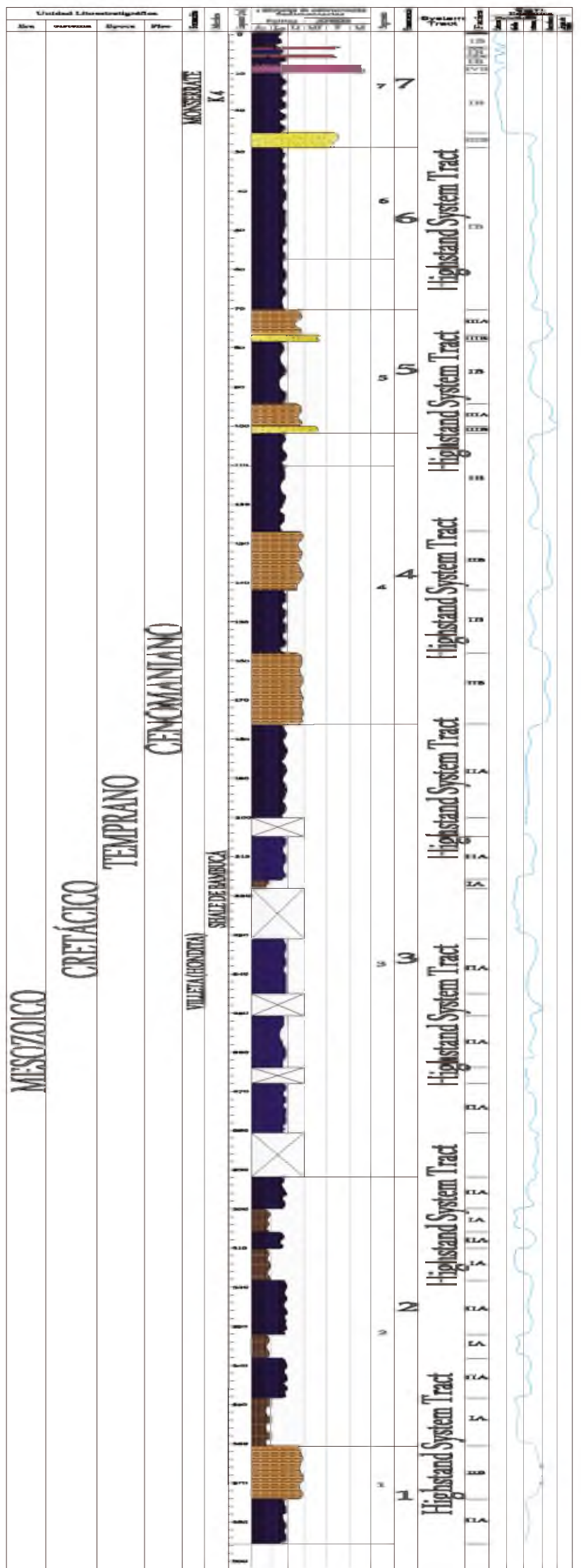

Figura 6.

Columna estratigráfica para Los Yuyos.
Parasecuencia 3. Con un espesor de 25 metros, de limolitas, lodolitas, arcillolitas y areniscas. Esta parasecuencia representa una sucesión progradante de ambientes de plataforma interna a ambientes de plataforma externa.

Parasecuencia 2. Las estructuras sedimentarias poseen un espesor de 35 metros se presenta como un conjunto de lodolitas fisiles (shales) de color gris negruzco, la curva eustática se concluye que en esta parasecuencia predominan los ambientes de plataforma interna.

Parasecuencia 1. Comprende un espesor de 37.5 metros donde se presentan lodolitas grises oscuras más conocidos como shales y limolitas; reflejando en la curva eustática variación de ambientes de plataforma externa a ambientes de plataforma interna.

\section{Los Yuyos}

De acuerdo a los datos recogidos en campo, la poligonal abierta levantada de tope a base para el área de Los Yuyos tiene un recorrido de 1195.9 metros por el carreteable que conduce al valle erosionado por el rio Yaguaracito con 7 afloramientos, levantando una columna estratigráfica con 357.4 metros de espesor (Figura 6). En ella son característicos los afloramientos de lutitas con intercalaciones de arcillolitas, limolitas y areniscas de grano fino a muy fino.

\section{Transgressive System Tract}

Está conformado litológicamente por areniscas de grano fino a muy fino, paquetes de lodolitas y niveles de rocas fosfóricas representando un cambio en el sistema deposicional de plataforma interna a externa.

Parasecuencia 7. Se encuentra la base de la formación Monserrate representada en este segmento como la unidad K4 y se presenta como una secuencia de arcillolitas, limolitas, lodolitas y un nivel de roca fosfórica con un espesor promedio de unos 27.4 metros de espesor real. Esta parasecuencia predominan los ambientes de depositación de plataforma externa.

\section{Highstand System Tract}

La influencia activa de mareas y olas; dan a interpretar que estas superficies de baja energía fueron generadas durante un ascenso relativo del nivel del mar. El miembro Shale de Bambucá de la formación Villeta es generado por este ambiente de depositación. Teniendo en cuenta el resultado de los análisis litológicos de cada una de las muestras de roca y las 
estructuras sedimentarias indica que el nivel del mar presenta una elevación y por ende una profundización en la cuenca.

Parasecuencia 6. Se presenta como una secuencia de arcillolitas, limolitas, lodolitas y un nivel de roca fosfórica con un espesor promedio de unos 27.4 metros de espesor real. Esta parasecuencia predominan los ambientes de depositación de plataforma externa.

Parasecuencia 5. Con un espesor real de 72.63 metros, está conformado por una secuencia de limolitas fisiles, lodolitas arenosas a limosas y hacia el tope las lodolitas se hacen más carbonosas. Esta parasecuencia los ambientes de sedimentación son plataforma interna.

Parasecuencia 4. Con un espesor real de 72.78 metros de toda la sección, de limolitas fisiles de color gris claro y lodolitas se presentan como niveles concresionales nodulares. El análisis facial a esta parasecuencia refleja una variación de ambientes de plataforma interna y shoreface.

Parasecuencia 3. Con un espesor de 10 metros esta sección se presentan un conjunto limolitas con estratificación media a fina, de color gris claro y de lodolitas de color gris claro, esta parasecuencia predominan los ambientes de depositación de plataforma interna.

Parasecuencia 2. balanceando las secciones por el método geométrico presenta un espesor real de 68,7 metros; una secuencia de arcillolitas con intercalaciones de lodolitas. El análisis de la curva eustática se concluye que en esta parasecuencia predominan los ambientes de plataforma media y plataforma interna.

Parasecuencia 1. Con un espesor de 26.3 metros y en contacto cubierto con las areniscas de la formación caballos superior se presenta una secuencia de lodolitas de color gris claro a amarillentas, muy fracturadas, fisiles y oxidadas. En esta sección se observan restos de fósiles de foraminíferos. Reflejando variación de ambientes de plataforma media a ambientes de plataforma interna.

\section{Conclusiones}

- El trabajo de estratigrafía en ambos puntos de estudio muestra que los Shales de Bambucá pertenecen a un ambiente de sedimentación de plataforma.
- El miembro Shale de Bambucá se encuentra en un sistema deposicional de tipo 2 (Highstand y Transgressive System Tract) que se caracteriza por la tasa desacelerante en el aumento del nivel del mar, dándole una menor movilidad al material arrastrado y por consiguiente, dándole características de laminación planoparalela con contenidos importantes de materia orgánica.

- Al tope de cada sección se encuentra un sistema deposicional similar, caracterizado por un aumento en el nivel del mar, un menor índice de depositación y un alto contenido de materia orgánica, seguido de una tasa máxima de aumento en el nivel del mar y un aumento en la acumulación de sedimentos, creando rocas sello y encapsulación de materia orgánica generadora de hidrocarburos.

- La recolección de muestras de lutitas representativas en los afloramientos contribuye en la investigación y análisis para determinar cantidad y tipo de materia orgánica, la capacidad que tiene la roca de aportar hidrocarburos y las características petrográficas.

- Las rocas del miembro Shale de Bambucá fueron asociadas al periodo Cretácico y las superficies de máxima inundación a los pisos Cenomaniano y Turoniano, donde se tiene mayor acumulación de materia orgánica y sedimentos.

- Este trabajo de grado además de tener carácter geológico, busca dar un enfoque adecuado para la recolección de muestras para la posterior realización de pruebas de laboratorio y análisis.

- El levantamiento y análisis estratigráfico junto a la recolección de muestras, determinan diferentes sucesos geológicos que acontecieron y que en el presente estructuran a la cuenca del Valle Superior del Magdalena y los campos petroleros que en ella han sido descubiertos.

\section{Referencias Bibliográficas}

1. Velandia, F., Núñez, A., Marquínez, G., 2001, Memoria explicativa para el departamento del Huila, INGEOMINAS.

2. Ferreira, P., Núñez, A., Rodríguez, M.A., 2002, Levantamiento geológico de la plancha 323, INGEOMINAS.

3. Marquínez, G., Morales, C. J., Caicedo, J.C., 2002, Memoria explicativa: plancha 344 Tesalia, INGEOMINAS.

4. Palencia, L.A., Chavarro, M.F., 2013, Correlación petrofísica en superficie con subsuelo de formaciones productoras subcuenca de Neiva, Universidad Surcolombiana, facultad de ingeniería de petróleos.

5. Peña, J.J., Annicchiarico, G.P., Jaramillo, J.M., Velazquez, E., 2002, Las calizas de Tetuán: una unidad 
litoestratigráfica para la subcuenca de Neiva, Valle Superior del Magdalena. Estratigrafia, petrografia y ambiente sedimentario. Revista Académica Colombiana de Ciencia. Volumen XXVI, número 101.

6. Martínez Álvarez, J. A., 1980. Mapas Geológicos Explicación e interpretación, PARANINFO, S. A., $2^{\mathrm{a}}$. Edición.

7. Porta, J. 1965. "Estratigrafía del Cretácico Superior y Terciario en el extremo sur del Valle Medio del Magdalena". UIS, Bol. Geol., 19:1-50.

8. Patarroyo, P., 1993. "Las formaciones cretácicas Hondita y Loma Gorda a Propósito de la nomenclatura estratigráfica del Valle Superior del Magdalena, Colombia". Trabajo presentado al VI Congreso Colombiano de Geología.

9. Sarmiento, L.F., Rangel, A., 2001, "Sistemas de Petróleo del Valle Superior del Magdalena, Colombia.", Ecopetrol.
10. Pérez, R., Perdomo J., 2013, Metodología para la caracterización de yacimientos no convencionales de gas shale en Colombia-VIM, Universidad Surcolombiana, facultad de ingeniería de petróleos.

11. Duvel et al, 1992. Types an hierarchies of stratigraphic cycles, Abstract Mesozoic and Cenozoic sequence stratigraphy of European basins Symposium, Dyjon.

12. Vargas, R., 2002. Proyecto de Integración Cartográfica de la Geología de la Cordillera Central y Occidental de Colombia Aplicada a la Exploración Aurífera, Geotec Ltda. Anglo Gold Ashanti Colombia.

13. Vargas, R., 2010, Estratigrafía del Jurásico de la Región Surcolombiana, USCO.

14. Vargas, R., 2013, Reconocimiento Geológico del Basamento Económico de la subcuenca de Neiva y su perspectiva como yacimientos no convencionales. Universidad Surcolombiana. 